Bangladesh Rice J. 19(1):63 - 71, 2015

\title{
Development and Validation of Producer and Consumer Preference Models for Rice Varieties in Bangladesh
}

\author{
Md Ismail Hossain ${ }^{1}$, Niaz Md Farhat Rahman ${ }^{1 *}$, Md Shahjahan Kabir ${ }^{2}$, Md Abu Bakr Siddique ${ }^{3}$ \\ and Muhammad Tareq ${ }^{4}$
}

\begin{abstract}
Rice production depends on both producers' and consumers' preference. The consumption of rice depends on consumers' taste and habits. The objectives of this study were to develop and validate mathematical models for producers', consumers' and producers-cum-consumers' preference to rice varieties and to evaluate the factors affecting both producers' and producer-cum-consumers' decision on varieties for rice cultivation and can provide an indication of the factors affecting consumers' preferences to rice varieties in Dhaka, Gazipur, Dinajpur and Bhola districts of Bangladesh. Chi-square $\left(\chi^{2}\right)$ tests were used to explore the significant difference of preferring rice varieties among the groups of people and compared the results with the proposed models for validation. Producers and producers-cum-consumers preferred BR11, BR22 and BRRI dhan32 in T. Aman; BR16, BRRI dhan28 and BRRI dhan29 in Boro and BR9, BR16 and BR20 in Aus seasons respectively. The specific grain quality characteristics such as whiteness, brokens, shape, amylose (\%), aroma, cooking quality, hardness and chalkiness influenced the consumers and producers preference. Furthermore, pure consumers also preferred rice varieties on the basis of its tastiness and fineness.
\end{abstract}

Keywords: Rice varieties, consumer, producer, producer-cum-consumer and preferences

\section{INTRODUCTION}

Rice, the staple food in Bangladesh, occupies nearly $90 \%$ of the total net cropped area of the country and more than $99 \%$ of the people eat rice as their main food @ 416 gm/person/day (HIES, 2010). Bangladesh Rice research Institute (BRRI) varieties occupying $80 \%$ of the total rice area account for $90 \%$ of the total rice production (Annual Report, BRRI, 2010-11). Crop agriculture in Bangladesh is mainly characterised by a rice monoculture. Almost $80 \%$ of the total cropped area is planted with rice, which accounts for more than $90 \%$ of total grain production (Alauddin and Tisdell, 1987; BBS, 2009; Asaduzzaman et al., 2010). The percentage share of rice in value term is more than $60 \%$ of the total crop agriculture (Asaduzzaman et al., 2010; Yu et al., 2010). The production of rice depends on both producers' and consumers' preference. It varies from variety to variety and the rice consumption depends on consumers' preference. Usually they were very concerned about the quality and price of the commodity when they made a purchase (Diako et al., 2010). In America consumers preferred rice, which is associated with specific cooking types and menu as well as the processing characteristics. Whereas, in the Middle East they mostly favoured a long grain and well-milled rice with strong aroma compared to the Europeans who tend to prefer the long grain with no scent. The Japanese, on the other hand, put high priority on the well-milled, very recently processed, shortgrain Japonica rice. The well-milled and long grain Indica rice, however, was preferred by consumers in Thailand (Lancon et al., 2003, Galawat and Yabe, 2010, Suwannaporn and Linnamenn, 2008). In contrast, the imported rice became a consumer choice in Nigeria due to their cleanliness and swelling capacity, taste, availability and grain shape. These characteristics of imported rice also are mostly preferred by restaurants and fast food industries to be used in their businesses. (Akaeze, 2010).

Suwannaporn and Linnemann (2008) and Basorun (2008) reported that consumers in Japan, Korea, North China and Taiwan, prefer to purchase rice at low price. There were increasing demands for local rice in these countries as the price was cheaper than the imported one. High price is a factor that prevents consumers to purchase certain rice brand that they preferred. The dominance of the demographic factors was also highlighted in a number of studies.

\footnotetext{
${ }^{1}$ Agricultural Statistics Division, ${ }^{2}$ Director (Administration and Common Service), ${ }^{3}$ Agricultural Economics Division, Bangladesh Rice Research Institute, Gazipur 1701, ${ }^{4}$ Department of Statistics, Jagannath University, Dhaka, *Corresponding author's E-mail: niaz.stat@brri.gov.bd
} 
Consumers who lived in urban area and had high standard of living, high income and education, tend to purchase rice of high quality based on their nutritional content (Tomlins et al., 2005). Whereas Kassali et al. (2010) found that income, age of the consumers and frequency of purchase were the important factors that influence household food consumption. Consumers tend to make a purchase at the retails closer to their homes because it makes them easier to get the rice and will purchase whichever rice brand that is available in the market. (Azabagaoglu and Gaytancioglu, 2009).The consumers in Brunei tend to purchase an imported rice rather than local one due to its availability in the market and family inherent where consumers have been using imported rice since childhood (Galawat and Yabe, 2010).

Wong et al. (2010) suggested that in future, in order to cope with the demand and changing lifestyle and consumer preference, rice should be made available in different forms: pre-cooked or instant rice, easy-to-cook and ready meal, and various packaging. It was also reported that changes in life style and time constraints on women would also affect the purchasing behaviour on rice brands in the market. Some of them may prefer to eat outside rather than preparing cook at home (Abdullah Farah et al., 2011). Thus, the study was undertaken with the objectives of examining the factors affecting producers' and consumers' preference for the rice varieties, their purchasing behaviour and to explore their consumption patterns.

\section{METHODOLOGY}

The study involved four districts - Gazipur and Dhaka as 'rice deficit' area and Dinajpur and Bhola as 'rice surplus' area. From each location fifty farmers were selected and the selected farmers were categorized into three groups such as: i) farmer as a producer; ii) farmer as a producer cum consumer; and iii) pure consumer (purchased rice from market). The pure consumers were selected from the urban areas of the selected districts. A pre-tested questionnaire was used for interviewing the farmers regarding choice of rice varieties for production and consumption. For comparison and validation 25 farmers' data were used from 50 farmers in the proposed models and remaining 25 farmers' data were used to explore the significant difference of preferring rice varieties among the group of people Chi-square $\left(\chi^{2}\right)$ tests (Gomez and Gomez, 1983) and survey on food conducted according to Larry McMullen (2004) were used to test the significant difference. The locations were savar upazila of Dhaka, Gazipur sadar of Gazipur, Fulbari upazila of Dinajpur and Bhola sadar of Bhola. In this study both male and female farmer and consumer were selected to get the variation of performance and effective result. Urban consumers pay high price for premium and fine rice compared to rural consumers may be because on average income of urban consumers is higher than the rural ones.

\section{Mathematical model for analysis}

The model of consumer demand for good characteristics is adapted from Ladd and Suvannunt (1976). Products and demand for the utility they provide, which in turn is a function of the characteristics of the product (Ladd and Suvannunt, p 505). Then Laurian J. Unnevehr (1986) has given a model of consumer demand for rice grain quality and return to research for quality improvement in southeast Asia. Also Juliano, B O (1982), presented a paper at food conference of Singapore institute of food science and technology about consumer acceptance and processing characteristics of rice varieties. On the basis of above authors model ideas; we proposed three models for consumer preference (Consumer model) and producer preference (Producer model) and producer-cum-consumer preference (Producer-Consumer model) to rice varieties.

\section{Consumer model}

$$
C P_{i}=f\left(P R_{j}, C I_{k}, C R V_{m}, A R V, V_{n}\right)
$$

where, $C P_{i}=$ Preference of $\mathrm{i}^{\text {th }}$ consumers; $P R_{j(1,2,3 \ldots)}=$ Price of rice; here $P R_{j}=\sum_{j=1}^{p} X_{R_{j}} R_{C_{j}}+U$; where,

$$
X_{R_{j}}=\text { Variance in the rice price; and } R_{C_{j}}=\sum_{j=1}^{p} P C_{j}+C C_{l}+O C_{k} ; P C_{j(\text { Whiteness,Brokens,Shape,Chalkiness })}=\text { Physical }
$$

characteristics of rice grain; $C C_{l(\text { amylose } \%), \text { Aroma })}=$ Chemical characteristics of rice grain;

$O C_{k(\text { cooking quality, eatingquality, Hardness) }}=$ Other characteristics; $C I_{k(H, M, L)}=\mathrm{k}^{\text {th }}$ consumer income;

$C R V_{m}=$ Category of rice variety; $A R V=$ Availability of rice variety and $\mathrm{V}_{\mathrm{n}}=$ Variety name.

$U=$ Constant variance (ie $E(U)=0$ ) 


\section{Producer model}

$P P_{i}=f\left(C P_{i}, P R V, R G E A E Z, T F S R_{s}, L F P, L D, R S S, T_{c}\right)$

where, $\quad P P_{i}=$ Preference of $\mathrm{i}^{\text {th }}$ producers; $C P_{i}=$ Preference of $\mathrm{i}^{\text {th }}$ consumers; $P R V=$ Production of rice variety (High production, Medium or average); RGEAEZ = Rice growing environment of AEZ; TFRS=Total farm size of rice, $L F P=$ Local farmer's practices; $L D=$ Local demand; $\mathrm{RSS}_{\mathrm{s}}=$ Rice seed source, $\mathrm{T}_{\mathrm{c}}=$ Traders category

\section{Producer-consumer model}

$P C_{l}=P P_{i}+C P_{i}$

where, $P P_{i}=$ Preference of $\mathrm{i}^{\text {th }}$ producers;

$C P_{i}=$ Preference of $\mathrm{i}^{\text {th }}$ consumers

\section{Data analysis}

The data were taken from the selected producers and consumers of Gazipur, Dhaka, Dinajpur and Bhola. Each sample was chosen randomly from producers and consumers. The retail markets were chosen to reflect the preference displayed by different income classes and price and variety name were recorded for each sample. In this study both qualitative and quantitative data were used. Qualitative data were analysed by descriptive statistics using ranking. The physical and chemical sample characteristics were analysed in the BRRI Grain Quality and Nutrition (GQN) Division's laboratory. The relationship of price and grain quality characteristics to rice is based on retail market demand and consumers' preference (Table 1). Tables 2, 3 and 4 respectively present the estimates of producers' preference, consumers' preference and producer-cumconsumer preference for the four locations. In four locations all parameters (variables), which introduced in the models are significant for producers' preference, consumers' preference and producer-cum-consumer preference.

\section{RESULTS AND DISCUSSION}

From the validation and analysis (linear regression) of the models and the $\chi^{2}$-test for the independence of preferring rice varieties among the selected group of respondents were significant in Dhaka area, which indicates that trend of preferring rice varieties differ from group to group (Tables 5 and 6). BR22 was highly preferred variety $(33 \%)$ in T. Aman season. Producer preference level was $28 \%$ and that of the producer-cum-consumers was $36 \%$.
In Boro season, BRRI dhan29 was highly preferred $(30 \%)$ variety. About $32 \%$ of the producer, $20 \%$ of the producer-cum-consumers and $34 \%$ of the pure consumers preferred this variety. In Aus season, BR20 was highly preferred $(43 \%)$ variety. About $34 \%$ of the producer as well as, $50 \%$ of the producer-cumconsumers preferred this variety.

In Gazipur area, the $\chi^{2}$-values for the independence of preferring rice varieties among the selected group of respondents were significant which indicates that trend of preferring rice varieties differ from group to group (Tables 5 and 7). BR11 was highly preferred $(52 \%)$ variety in T. Aman season. Producer $(50 \%)$ as well as producer-cumconsumers $(68 \%)$ equally preferred this variety. In Boro season, BRRI dhan 28 is highly preferred $(34 \%)$ variety. Twenty-eight percent of the producer, $32 \%$ of the producer-cum-consumers and $34 \%$ of the pure consumers preferred this variety. In Aus season, the preferred variety was BR16 (66\%). About $52 \%$ of the producer as well as, $66 \%$ of the producer-cum-consumers preferred this variety.

In Dinajpur area, $\chi^{2}$-values for preferring rice varieties vary significantly from group to group (Tables 5 and 8). In T. Aman season, the selected persons respond nothing about their variety choice. But in Boro season, the $\chi^{2}$-value indicates that trend of preferring rice varieties was more or less same in the selected group of people. The highest preferred variety in Boro season was BR16, where $30 \%$ producer and $24 \%$ producer-cum-consumer preferred this variety. Aus variety was not produced in the locality.

In Bhola area, the $\chi^{2}$-values for the independence of preferring rice varieties among the selected group of respondents were significant, which indicates that trend of preferring rice varieties differ from group to group (Tables 5 and 9). BR32 was highly preferred $(28 \%)$ variety in T. Aman season, where $26 \%$ producer and $30 \%$ producer-cum-consumers preferred this variety.

In Boro season, BRRI dhan29 was highly preferred $(25 \%)$ by the selected respondents. Twenty percent of the producers, $22 \%$ of the producer-cum-consumers and $34 \%$ of the pure consumers preferred this variety. In Aus season, BR9 was highly preferred (33\%) by the selected respondents. About $52 \%$ of the producer as well as, $66 \%$ of the producer-cum-consumers preferred this variety.

Tables 10, 11, 12 and 13 indicate that most of the selected persons, who are grouped as producer 
and producer-cum-consumer preferred rice varieties for their higher yield in Dhaka, Gazipur, Dinajpur and Bhola areas. On the other hand, the pure consumers preferred varieties based on their tastes (Table 14).

Table 1. Correlation of price and grain quality characteristics in four locations.

\begin{tabular}{|c|c|c|c|c|}
\hline \multirow[t]{2}{*}{ Characteristic } & \multicolumn{4}{|c|}{ Location } \\
\hline & Dhaka & Gazipur & Dinajpur & Bhola \\
\hline Price $(\mathrm{Tk} / \mathrm{kg})$ & 43.2 & 41.5 & 36.3 & 34.6 \\
\hline $\begin{array}{l}\text { (Average price both coarse and fine } \\
\text { rice) }\end{array}$ & $(6.7)^{\mathrm{c}}$ & $(6.1)$ & $(5.5)$ & $(5.1)$ \\
\hline \multicolumn{5}{|l|}{ Physical characteristics } \\
\hline Whiteness & 71.2 & 70.3 & 52.6 & 41.4 \\
\hline (\% of pure white/polish) & $(4.7)$ & $(4.4)$ & $(2.9)$ & $(2.5)$ \\
\hline Brokens & 15.6 & 16.2 & 20.7 & 30.8 \\
\hline (\% of grains) & $(14.9)$ & $(15.1)$ & $(11.6)$ & $(9.4)$ \\
\hline Shape & 3.5 & 3.3 & 2.5 & 2.1 \\
\hline (length and width) & $(0.3)$ & $(0.3)$ & $(0.2)$ & $(0.2)$ \\
\hline \multicolumn{5}{|l|}{ Chemical characteristics } \\
\hline \multirow[t]{2}{*}{ Amylose $(\%)$} & 27.9 & 26.7 & 25.3 & 25.8 \\
\hline & $(2.6)$ & (2.5) & $(2.2)$ & $(2.8)$ \\
\hline Aroma & 5.36 & 5.12 & 2.45 & 2.10 \\
\hline (No aroma, less aroma, strong aroma) & $(0.98)$ & $(0.92)$ & $(1.56)$ & $(1.48)$ \\
\hline Cooking quality & 7.1 & 7.3 & 10.4 & 10.6 \\
\hline (Time) & $(0.7)$ & $(0.8)$ & $(0.5)$ & $(0.5)$ \\
\hline Hardness & 1.1 & 1.3 & 1.7 & 1.9 \\
\hline (Sticky or non-sticky) & $(0.20)$ & $(0.22)$ & $(0.41)$ & $(0.49)$ \\
\hline Eating quality & 6.43 & 6.46 & 7.32 & 7.42 \\
\hline (taste) & $(1.16)$ & $(1.18)$ & $(1.34)$ & $(1.36)$ \\
\hline \multicolumn{5}{|l|}{ Varietal characteristics } \\
\hline Chalkiness & 5.5 & 5.8 & 6.2 & 6.6 \\
\hline (proportion of grain) & (3.1) & (3.3) & $(4.1)$ & $(4.3)$ \\
\hline No. of samples & 25 & 25 & 25 & 25 \\
\hline $\mathrm{R}^{2}$ & 0.89 & 0.82 & 0.73 & 0.69 \\
\hline
\end{tabular}

CStandard deviations are in parenthesis.

Table 2. Regression estimate of producers preference in four locations.

\begin{tabular}{l|c|c|c|c|c|c|c|c|c}
\hline Location & No. of sample & Intercept & $\mathrm{PR}_{\mathrm{j}}$ & $\mathrm{CI}_{\mathrm{k}}$ & $\mathrm{CRV}_{\mathrm{m}}$ & $\mathrm{ARV}$ & $\mathrm{V}_{\mathrm{n}}$ & $\mathrm{R}^{2}$ & $\begin{array}{l}\text { Adjusted } \\
\mathrm{R}^{2}\end{array}$ \\
\hline Dhaka & 25 & 10.36 & $0.58^{* *}$ & $0.21^{*}$ & $0.052^{*}$ & $0.043^{*}$ & $0.047^{*}$ & 0.85 & 0.81 \\
Gazipur & 25 & 10.22 & $0.59^{* *}$ & $0.20^{*}$ & $0.056^{*}$ & $0.042^{*}$ & $0.045^{*}$ & 0.87 & 0.78. \\
Dinajpur & 25 & 11.30 & $0.56^{* *}$ & $0.24^{*}$ & $0.052^{*}$ & $0.044^{*}$ & $0.042^{*}$ & 0.79 & 0.77 \\
Bhola & 25 & 11.15 & $0.55^{* *}$ & $0.22^{*}$ & $0.051^{*}$ & $0.040^{*}$ & $0.043^{*}$ & 0.75 & 0.76 \\
\hline
\end{tabular}

Note: ** and * denotes significant at the $1 \%$ and the $5 \%$ level respectively.

Table 3. Regression estimate of consumers preference in four locations.

\begin{tabular}{c|c|c|c|c|c|c|c|c|c|c|c|c}
\hline Location & $\begin{array}{l}\text { No. of } \\
\text { sample }\end{array}$ & Intercept & CP $_{\mathrm{i}}$ & PRV & RGEAEZ & TFSR & SRS & LEP & LD & TC $_{C}$ & $\begin{array}{c}\mathrm{R}^{2} \\
\text { Adjusted } \\
\mathrm{R}^{2}\end{array}$ \\
\hline Dhaka & 25 & 30.28 & $0.61^{* *}$ & $0.39^{*}$ & $0.047^{*}$ & $0.019^{*}$ & $0.013^{*}$ & $0.033^{*}$ & $0.035^{*}$ & $0.033^{*}$ & 0.87 & 0.82 \\
Gazipur & 25 & 30.47 & $0.63^{* *}$ & $0.36^{*}$ & $0.046^{*}$ & $0.014^{*}$ & $0.011^{*}$ & $0.030^{*}$ & $0.037^{*}$ & $0.031^{*}$ & 0.85 & 0.81 \\
Dinajpur & 25 & 28.36 & $0.59^{* *}$ & $0.34^{*}$ & $0.044^{*}$ & $0.012^{*}$ & $0.012^{*}$ & $0.029^{*}$ & $0.032^{*}$ & $0.034^{*}$ & 0.83 & 0.79 \\
Bhola & 25 & 27.93 & $0.55^{* *}$ & $0.33^{*}$ & $0.040^{*}$ & $0.011^{*}$ & $0.014^{*}$ & $0.028^{*}$ & $0.030^{*}$ & $0.031^{*}$ & 0.81 & 0.77 \\
\hline
\end{tabular}

Note: ** and * denotes significant at $1 \%$ and $5 \%$ level respectively.

Table 4. Regression estimate of Producer-cum-consumers' preference in four locations.

\begin{tabular}{lc|c|c|c|c|c}
\hline \multicolumn{1}{c}{ Location } & No. of sample & Intercept & $\mathrm{PP}_{\mathrm{i}}$ & $\mathrm{CP}_{\mathrm{i}}$ & $\mathrm{R}^{2}$ & Adjusted $\mathrm{R}^{2}$ \\
\hline Dhaka & 25 & 58.68 & $0.55^{* *}$ & $0.44^{* *}$ & 0.89 & 0.78 \\
Gazipur & 25 & 57.76 & $0.53^{* *}$ & $0.43^{* *}$ & 0.87 & 0.73 \\
Dinajpur & 25 & 55.39 & $0.51^{* *}$ & $0.40^{* *}$ & 0.86 & 0.71 \\
Bhola & 25 & 54.67 & $0.50^{* *}$ & $0.41^{* *}$ & 0.84 & 0.69 \\
\hline
\end{tabular}

Note: ${ }^{* *}$ denotes significant at the $1 \%$ level. 
Table 5. Combined analysis of proposed regression models.

Consumer model: $\mathrm{CP}_{\mathrm{i}}=10.76+0.59 \mathrm{PR}_{\mathrm{j}}^{* *}+0.24 \mathrm{CI}_{\mathrm{k}}{ }^{*}+0.55 \mathrm{CRV}_{\mathrm{m}}{ }^{*}+0.043 \mathrm{ARV}^{*}+0.48 \mathrm{~V}_{\mathrm{n}}{ }^{*} ; \mathrm{R}^{2}=0.82$

Producer model: $\mathrm{PP}_{\mathrm{i}}=30.11+0.66 \mathrm{CP}_{\mathrm{i}}^{* *}+0.035 \mathrm{PRV}^{*}+0.046$ RGEAEZ $^{*}+0.018 \mathrm{TFSR}^{*}+0.015 \mathrm{SRS}^{*}+0.029 \mathrm{LEP}^{*}+$ $0.033 \mathrm{LD}^{*}+0.031 \mathrm{TC}^{*}$

$\mathrm{R}^{2}=0.84$

Producer-cum-consumer model: $\mathrm{PC}_{1}=56.84+0.51 \mathrm{PP}_{\mathrm{i}}^{* *}+0.40 \mathrm{CP}_{\mathrm{i}}^{* *} ; \mathrm{R}^{2}=0.88$

Note: ** and * denotes significant at the $1 \%$ and the $5 \%$ level respectively.

Table 6. Contingency table for the independence between group of variety and group of farmers on varietal preference in Dhaka area.

\begin{tabular}{|c|c|c|c|c|c|c|c|}
\hline \multirow{2}{*}{$\begin{array}{l}\text { Season } \\
\text { Group/Variety }\end{array}$} & \multicolumn{6}{|c|}{ T. Aman } & \multirow[b]{2}{*}{ Total } \\
\hline & BR11 & BR22 & $\begin{array}{c}\text { BRRI } \\
\text { dhan32 }\end{array}$ & \begin{tabular}{c|} 
BRRI \\
dhan40
\end{tabular} & Chinigura & Non-respondent & \\
\hline Producer & $3(12)$ & $7(28)$ & $5(20)$ & $2(8)$ & $4(16)$ & $4(16)$ & $25(100)$ \\
\hline $\begin{array}{l}\text { Producer-cum- } \\
\text { consumer }\end{array}$ & $2(8)$ & $9(36)$ & $6(24)$ & $3(12)$ & $2(8)$ & $3(12)$ & $25(100)$ \\
\hline Pure consumer & $4(16)$ & $8(32)$ & $6(24)$ & $4(16)$ & $1(4)$ & $2(8)$ & $25(100)$ \\
\hline Total & $9(12)$ & $24(32)$ & $17(23)$ & $9(12)$ & $7(9)$ & $9(12)$ & $75(100)$ \\
\hline Chi-square value & \multicolumn{7}{|c|}{$13.243^{*}$} \\
\hline Season & \multicolumn{6}{|c|}{ Boro } & \multirow[b]{2}{*}{ Total } \\
\hline Group/Variety & BR16 & BRRI dhan28 & $\begin{array}{l}\text { BRRI } \\
\text { dhan29 }\end{array}$ & $\begin{array}{l}\text { BRRI } \\
\text { dhan47 }\end{array}$ & Savayra Sail & $\begin{array}{c}\text { Non- } \\
\text { respondent }\end{array}$ & \\
\hline Producer & $3(12)$ & $5(20)$ & $8(32)$ & $2(8)$ & $4(16)$ & $3(12)$ & $25(100)$ \\
\hline $\begin{array}{l}\text { Producer-cum- } \\
\text { consumer }\end{array}$ & $4(16)$ & $4(16)$ & $6(24)$ & $2(8)$ & $5(20)$ & $4(16)$ & $25(100)$ \\
\hline Pure consumer & $3(12)$ & $3(12)$ & $9(36)$ & $3(12)$ & $5(20)$ & $2(8)$ & $25(100)$ \\
\hline Total & $10(13)$ & $12(16)$ & $23(31)$ & $7(9)$ & $14(19)$ & $9(12)$ & $75(100)$ \\
\hline Chi-square value & \multicolumn{7}{|c|}{$21.364^{* *}$} \\
\hline Season & \multicolumn{6}{|c|}{ Aus } & \multirow[b]{2}{*}{ Total } \\
\hline Group/Variety & BR1 & BR20 & $\begin{array}{l}\text { BRRI } \\
\text { dhan26 }\end{array}$ & $\begin{array}{c}\text { BRRI } \\
\text { dhan27 }\end{array}$ & Pueikka & $\begin{array}{l}\text { Non- } \\
\text { respondent }\end{array}$ & \\
\hline Producer & $4(16)$ & $8(32)$ & $3(12)$ & $1(4)$ & $4(16)$ & $5(20)$ & $25(100)$ \\
\hline $\begin{array}{l}\text { Producer-cum- } \\
\text { consumer }\end{array}$ & $2(8)$ & $11(44)$ & $4(16)$ & $2(8)$ & $3(12)$ & $3(12)$ & $25(100)$ \\
\hline Pure consumer & $3(12)$ & $12(48)$ & $2(8)$ & $1(2)$ & $4(16)$ & $3(12)$ & $25(100)$ \\
\hline Total & $9(12)$ & $31(41)$ & $9(12)$ & $4(5)$ & $11(15)$ & $11(15)$ & $75(100)$ \\
\hline
\end{tabular}

\begin{tabular}{l|l} 
Chi-square value & $5.431(\mathrm{NS})$ \\
\hline Note: Figures in the parentheses represent percentage, ${ }^{* *}=$ significant at the $1 \%$ level, ${ }^{*}=$ significant at the $5 \%$ level, NS
\end{tabular}

Note. Figures in the parentheses represent percentage, ${ }^{2}$ significant at the $1 \%$ level, ${ }^{2}$ significant at the $5 \%$ level, NS

Table 7. Contingency table for the independence between group of variety and group of farmers on varietal preference in Gazipur area.

\begin{tabular}{|c|c|c|c|c|c|c|}
\hline Season & \multicolumn{5}{|c|}{ T. Aman } & \multirow[b]{2}{*}{ Total } \\
\hline Group/Variety & BR3 & BR11 & BR22 & BR25 & $\begin{array}{c}\text { Non- } \\
\text { respondent }\end{array}$ & \\
\hline Producer & $1(4)$ & $12(48)$ & \multicolumn{2}{|l|}{$2(8)$} & $9(36)$ & $25(100)$ \\
\hline Producer-cum-consumer & $2(8)$ & $16(64)$ & $1(4)$ & $1(4)$ & $5(20)$ & $25(100)$ \\
\hline Pure consumer & $4(16)$ & $8(32)$ & $8(32)$ & $4(16)$ & $1(2)$ & $25(100)$ \\
\hline Total & $7(9)$ & $36(48)$ & \multirow[t]{2}{*}{$11(15)$} & $6(8)$ & $15(20)$ & $75(100)$ \\
\hline Chi-square value & \multicolumn{4}{|l|}{$16.291^{*}$} & & \\
\hline Significance level & \multicolumn{5}{|l|}{0.050} & \\
\hline Season & \multicolumn{5}{|c|}{ Boro } & \multirow{2}{*}{ Total } \\
\hline Group/Variety & BR14 & $\begin{array}{c}\text { BRRI } \\
\text { dhan28 }\end{array}$ & $\begin{array}{c}\text { BRRI } \\
\text { dhan29 }\end{array}$ & $\begin{array}{c}\text { BRRI } \\
\text { Dhan36 }\end{array}$ & $\begin{array}{c}\text { Non- } \\
\text { respondent }\end{array}$ & \\
\hline Producer & $1(4)$ & $7(28)$ & $8(32)$ & $2(8)$ & $7(28)$ & $25(100)$ \\
\hline Producer-cum-consumer & $1(4)$ & $8(32)$ & $9(36)$ & $2(8)$ & $5(20)$ & $25(100)$ \\
\hline Pure consumer & $3(12)$ & $10(40)$ & $7(28)$ & $3(12)$ & $2(8)$ & $25(100)$ \\
\hline Total & $5(7)$ & 25(33) & $24(32)$ & $7(9)$ & $14(19)$ & $75(100)$ \\
\hline Chi-square value & $25.488^{* *}$ & & & & & \\
\hline Significance level & 0.002 & & & & & \\
\hline Season & & & Aus & & & \\
\hline
\end{tabular}


Bangladesh Rice J. 19(1):63 - 71, 2015

\begin{tabular}{|c|c|c|c|c|c|}
\hline Group/Variety & BR3 & BR16 & Kalijira & Non-respondent & Total \\
\hline Producer & $5(20)$ & $13(52)$ & $1(4)$ & $6(24)$ & $25(100)$ \\
\hline Producer-cum-consumer & $2(8)$ & $16(64)$ & $3(12)$ & $4(16)$ & $25(100)$ \\
\hline Pure consumer & $5(20)$ & $20(80)$ & 0 & 0 & $25(100)$ \\
\hline Total & $12(16)$ & $49(65)$ & $4(5)$ & $10(13)$ & 75(100) \\
\hline Chi-square value & $6.875(\mathrm{NS})$ & & & & \\
\hline Significance level & 0.537 & & & & \\
\hline
\end{tabular}


Bangladesh Rice J. 19(1):63 - 71, 2015

Note: Figures in the parentheses represent percentage, ${ }^{* *}=$ significant at the $1 \%$ level, ${ }^{*}=$ significant at the $5 \%$ level, NS =Not significant.

Table 8. Contingency table for the independence between group of variety and group of farmers on varietal preference in Dinajpur area.

\begin{tabular}{|c|c|c|c|c|c|c|c|c|c|c|c|c|}
\hline Season & \multicolumn{11}{|c|}{ T. Aman } & \multirow{2}{*}{ Total } \\
\hline $\begin{array}{l}\text { Group/Variet } \\
\text { y }\end{array}$ & \multicolumn{2}{|c|}{ BR11 } & BR22 & & $\begin{array}{l}\text { utisharn } \\
\text { a }\end{array}$ & \multicolumn{2}{|c|}{ Ranjit } & Jatapari & \multicolumn{3}{|c|}{ Non-respondent } & \\
\hline Producer & \multicolumn{2}{|c|}{$2(8)$} & $3(12)$ & \multicolumn{2}{|r|}{$2(8)$} & \multicolumn{2}{|l|}{$2(8)$} & $4(16)$ & \multicolumn{3}{|c|}{$12(48)$} & $25(100)$ \\
\hline $\begin{array}{l}\text { Producer-cum- } \\
\text { consumer }\end{array}$ & \multicolumn{2}{|c|}{$4(16)$} & $1(4)$ & \multicolumn{2}{|r|}{$2(8)$} & \multicolumn{2}{|l|}{$1(4)$} & $1(2)$ & \multicolumn{3}{|c|}{$16(64)$} & $25(100)$ \\
\hline Pure consumer & \multicolumn{2}{|c|}{$6(24)$} & $4(16)$ & \multirow{2}{*}{\multicolumn{2}{|c|}{$\begin{array}{l}1(4) \\
5(7)\end{array}$}} & \multicolumn{2}{|l|}{$1(4)$} & $3(12)$ & \multicolumn{3}{|c|}{$10(40)$} & $25(100)$ \\
\hline Total & \multicolumn{2}{|c|}{$12(16)$} & $8(11)$ & & & \multicolumn{2}{|l|}{$4(5)$} & $8(11)$ & \multicolumn{3}{|c|}{$38(50)$} & $75(100)$ \\
\hline $\begin{array}{l}\text { Chi-square } \\
\text { value }\end{array}$ & \multicolumn{12}{|c|}{$44.392^{* *}$} \\
\hline $\begin{array}{l}\text { Significance } \\
\text { level }\end{array}$ & \multicolumn{12}{|l|}{0.000} \\
\hline Season & \multicolumn{11}{|c|}{ Boro } & $\begin{array}{c}\text { Tota } \\
1\end{array}$ \\
\hline $\begin{array}{l}\text { Group/Variet } \\
\text { y }\end{array}$ & $\begin{array}{c}\text { BR } \\
1\end{array}$ & BR16 & $\begin{array}{r}\text { BR } \\
\text { dha }\end{array}$ & & $\begin{array}{c}\text { BRRI } \\
\text { dhan29 }\end{array}$ & $\begin{array}{c}\text { Gutisharn } \\
\text { a }\end{array}$ & $\begin{array}{l}\text { Jata } \\
\text { Pari }\end{array}$ & $\begin{array}{c}\text { Hybri } \\
\text { d }\end{array}$ & $\begin{array}{c}\text { Chin } \\
\text { a }\end{array}$ & $\begin{array}{c}\text { Parij } \\
\text { a }\end{array}$ & $\begin{array}{c}\text { Non- } \\
\text { responde } \\
\text { nt }\end{array}$ & \\
\hline Producer & $1(4)$ & $4(16)$ & $4(1$ & & $1(4)$ & $4(16)$ & $2(8)$ & $3(12)$ & $1(4)$ & $1(4)$ & $4(16)$ & $\begin{array}{c}25 \\
(100)\end{array}$ \\
\hline $\begin{array}{l}\text { Producer-cum- } \\
\text { consumer }\end{array}$ & $1(4)$ & $6(24)$ & $5(2$ & & $1(4)$ & $2(8)$ & $3(12)$ & $3(12)$ & $1(4)$ & 0 & $3(12)$ & $\begin{array}{c}25 \\
(100)\end{array}$ \\
\hline Pure consumer & $1(4)$ & $2(8)$ & $4(1$ & & $3(12)$ & $1(4)$ & $7(28)$ & $3(12)$ & $0(0)$ & $3(12)$ & $1(4)$ & $\begin{array}{c}25 \\
(100)\end{array}$ \\
\hline Total & $3(4)$ & $\begin{array}{c}12(16 \\
)\end{array}$ & 13( & & $5(7)$ & $7(9)$ & $12(16)$ & $9(12)$ & $2(3)$ & $4(5)$ & $8(11)$ & $\begin{array}{c}75 \\
(100)\end{array}$ \\
\hline $\begin{array}{l}\text { Chi-square } \\
\text { value }\end{array}$ & 12.276 & (NS) & & & & & & & & & & \\
\hline $\begin{array}{l}\text { Significance } \\
\text { level }\end{array}$ & 0.764 & & & & & & & & & & & \\
\hline
\end{tabular}


Bangladesh Rice J. 19(1):63 - 71, 2015

Table 9. Contingency table for the independence between group of variety and group of farmers on varietal preference in Bhola area.

\begin{tabular}{|c|c|c|c|c|c|c|c|}
\hline \multirow{2}{*}{$\begin{array}{l}\text { Season } \\
\text { Group/Variety }\end{array}$} & \multicolumn{6}{|c|}{ T. Aman } & \multirow[b]{2}{*}{ Total } \\
\hline & BR11 & $\begin{array}{c}\text { BRRI } \\
\text { dhan32 }\end{array}$ & dhan40 & Mota & Kajal Sail & $\begin{array}{c}\text { Non- } \\
\text { respondent }\end{array}$ & \\
\hline Producer & $6(24)$ & $6(24)$ & $4(16)$ & $3(12)$ & \multicolumn{2}{|r|}{$1(4)$} & $25(100)$ \\
\hline $\begin{array}{l}\text { Producer-cum- } \\
\text { consumer }\end{array}$ & $5(20)$ & $7(28)$ & $2(4)$ & $5(20)$ & \multicolumn{2}{|l|}{$5(20)$} & $25(100)$ \\
\hline Pure consumer & $7(28)$ & $7(28)$ & $1(4)$ & $6(24)$ & \multicolumn{2}{|l|}{$2(8)$} & $25(100)$ \\
\hline Total & $18(24)$ & $20(27)$ & $7(9)$ & $14(19)$ & \multicolumn{2}{|l|}{$12(16)$} & $75(100)$ \\
\hline Chi-square value & \multicolumn{7}{|l|}{$13.457^{*}$} \\
\hline Significance level & \multicolumn{7}{|l|}{0.050} \\
\hline Season & \multicolumn{6}{|c|}{ Boro } & \multirow[b]{2}{*}{ Total } \\
\hline Group / Variety & $\begin{array}{c}\text { BRRI } \\
\text { dhan28 }\end{array}$ & $\begin{array}{c}\text { BRRI } \\
\text { dhan29 }\end{array}$ & $\begin{array}{c}\text { BRRI } \\
\text { dhan47 }\end{array}$ & $\begin{array}{l}\text { Vojan } \\
\text { IRRI }\end{array}$ & Hybrid-2 & $\begin{array}{c}\text { Non- } \\
\text { respondent }\end{array}$ & \\
\hline Producer & $3(12)$ & $5(20)$ & $\begin{array}{l}\text { dhan47 } \\
\text { 4(16) }\end{array}$ & $6(24)$ & $5(20)$ & $2(8)$ & $25(100)$ \\
\hline $\begin{array}{l}\text { Producer-cum- } \\
\text { consumer }\end{array}$ & $4(16)$ & $6(24)$ & $4(16)$ & $4(16)$ & \multicolumn{2}{|l|}{$4(16)$} & $25(100)$ \\
\hline Pure consumer & $5(20)$ & $8(32)$ & $2(8)$ & $5(20)$ & \multicolumn{2}{|l|}{$3(12)$} & $25(100)$ \\
\hline Total & 12(16) & $19(26)$ & $10(13)$ & $15(20)$ & \multicolumn{2}{|l|}{$12(16)$} & $150(100)$ \\
\hline Chi-square value & \multicolumn{7}{|l|}{$21.217^{* *}$} \\
\hline Significance level & \multicolumn{7}{|l|}{0.002} \\
\hline Season & \multicolumn{6}{|c|}{ Aus } & \multirow[b]{2}{*}{ Total } \\
\hline Group/Variety & BR8 & & & BR24 & Kali hytta & $\begin{array}{c}\text { Non- } \\
\text { respondent }\end{array}$ & \\
\hline \multirow{2}{*}{$\begin{array}{l}\text { Producer } \\
\text { Producer-cum- } \\
\text { consumer }\end{array}$} & $4(16)$ & \multicolumn{2}{|c|}{$8(32)$} & $4(16)$ & \multicolumn{2}{|r|}{$4(16)$} & $25(100)$ \\
\hline & $4(14)$ & \multicolumn{2}{|c|}{$7(28)$} & $4(16)$ & $5(20)$ & $5(20)$ & $50(100)$ \\
\hline Pure consumer & $5(20)$ & \multicolumn{2}{|c|}{$9(36)$} & $1(4)$ & $6(24)$ & $4(16)$ & $50(100)$ \\
\hline Total & 13(17) & & & $9(12)$ & $16(22)$ & $13(17)$ & $75(100)$ \\
\hline Chi-square value & $3.198^{*}$ & & & & & & \\
\hline Significance level & 0.030 & & & & & & \\
\hline
\end{tabular}

Table 10. Liking reasons for BRRI varieties in Dhaka.

\begin{tabular}{|c|c|c|c|c|c|c|c|c|c|c|c|c|}
\hline Season & \multicolumn{4}{|c|}{ T. Aman } & \multicolumn{4}{|c|}{ Boro } & \multicolumn{4}{|c|}{ Aus } \\
\hline Comment/Variety & BR11 & BR22 & BR32 & BR40 & BR16 & $\begin{array}{c}\text { BRRI } \\
\text { dhan28 }\end{array}$ & $\begin{array}{c}\text { BRRI } \\
\text { dhan29 }\end{array}$ & $\begin{array}{c}\text { BRRI } \\
\text { dhan47 }\end{array}$ & BR1 & BR20 & $\begin{array}{c}\text { BRRI } \\
\text { dhan26 }\end{array}$ & $\begin{array}{c}\text { BRRI } \\
\text { dhan27 }\end{array}$ \\
\hline Higher yield & $\begin{array}{c}5 \\
(56)\end{array}$ & $\begin{array}{c}9 \\
(41)\end{array}$ & $\begin{array}{c}11 \\
(44)\end{array}$ & $\begin{array}{c}4 \\
(44)\end{array}$ & $\begin{array}{l}10 \\
(72)\end{array}$ & $\begin{array}{c}10 \\
(56)\end{array}$ & $\begin{array}{c}11 \\
(44)\end{array}$ & $\begin{array}{c}4 \\
(57)\end{array}$ & $\begin{array}{c}7 \\
(62)\end{array}$ & $\begin{array}{c}10 \\
(40)\end{array}$ & $\begin{array}{c}4 \\
(28)\end{array}$ & $\begin{array}{c}2 \\
(40)\end{array}$ \\
\hline Tasty & $\begin{array}{c}4 \\
(44)\end{array}$ & $\begin{array}{c}13 \\
(59)\end{array}$ & $\begin{array}{c}12 \\
(48)\end{array}$ & $\begin{array}{c}5 \\
(56)\end{array}$ & $\begin{array}{c}2 \\
(14)\end{array}$ & $\begin{array}{c}4 \\
(22)\end{array}$ & $\begin{array}{c}10 \\
(40)\end{array}$ & - & $\begin{array}{c}5 \\
(38)\end{array}$ & $\begin{array}{c}10 \\
(40)\end{array}$ & $\begin{array}{c}5 \\
(36)\end{array}$ & $\begin{array}{c}2 \\
(40)\end{array}$ \\
\hline $\begin{array}{l}\text { Tasty +less } \\
\text { production cost }\end{array}$ & - & - & $\begin{array}{c}2 \\
(8)\end{array}$ & - & - & - & - & $\begin{array}{c}3 \\
(43)\end{array}$ & & $\begin{array}{c}5 \\
(20)\end{array}$ & - & $\begin{array}{c}1 \\
(20)\end{array}$ \\
\hline $\begin{array}{l}\text { Testy + longer } \\
\text { durability of } \\
\text { boiled rice +less } \\
\text { nursing }\end{array}$ & - & - & - & - & $\begin{array}{c}2 \\
(14)\end{array}$ & $\begin{array}{c}2 \\
(11)\end{array}$ & $\begin{array}{c}2 \\
(8)\end{array}$ & & - & - & - & - \\
\hline Fine rice +tasty & - & - & - & - & - & - & - & - & - & - & $\begin{array}{c}5 \\
(36)\end{array}$ & - \\
\hline $\begin{array}{l}\text { Better yield +less } \\
\text { fertilizer use }\end{array}$ & - & - & - & - & - & $\begin{array}{c}2 \\
(11)\end{array}$ & $\begin{array}{c}2 \\
(8)\end{array}$ & - & - & - & & - \\
\hline Total & $\begin{array}{c}9 \\
(100)\end{array}$ & $\begin{array}{c}22 \\
(100)\end{array}$ & $\begin{array}{c}25 \\
(100)\end{array}$ & $\begin{array}{c}9 \\
(100)\end{array}$ & $\begin{array}{c}14 \\
(100)\end{array}$ & $\begin{array}{c}18 \\
(100)\end{array}$ & $\begin{array}{c}25 \\
(100)\end{array}$ & $\begin{array}{c}7 \\
(100)\end{array}$ & $\begin{array}{c}12 \\
(100)\end{array}$ & $\begin{array}{c}25 \\
(100)\end{array}$ & $\begin{array}{c}14 \\
(100)\end{array}$ & $\begin{array}{c}5 \\
(100)\end{array}$ \\
\hline
\end{tabular}

Note: Figures in the parentheses represent percentage. 
Bangladesh Rice J. 19(1):63 - 71, 2015

Table 11. Liking reasons for BRRI varieties in Gazipur.

\begin{tabular}{|c|c|c|c|c|c|c|c|c|c|c|c|}
\hline Season & \multicolumn{4}{|c|}{ T. Aman } & \multicolumn{4}{|c|}{ Boro } & \multicolumn{3}{|c|}{ Aus } \\
\hline Comment/Variety & BR3 & BR11 & BR22 & BR25 & BR14 & $\begin{array}{c}\text { BRRI } \\
\text { dhan28 }\end{array}$ & $\begin{array}{c}\text { BRRI } \\
\text { dhan29 }\end{array}$ & $\begin{array}{c}\text { BRRI } \\
\text { dhan36 }\end{array}$ & BR3 & BR16 & Kalijira \\
\hline Higher yield & $\begin{array}{c}2 \\
(65)\end{array}$ & $\begin{array}{c}12 \\
(48)\end{array}$ & $\begin{array}{c}3 \\
(75)\end{array}$ & $\begin{array}{c}2 \\
(50)\end{array}$ & $\begin{array}{c}2 \\
(40)\end{array}$ & $\begin{array}{c}23 \\
(92)\end{array}$ & $\begin{array}{c}12 \\
(50)\end{array}$ & $\begin{array}{c}3 \\
(50)\end{array}$ & $\begin{array}{c}8 \\
(62)\end{array}$ & $\begin{array}{l}16 \\
(64)\end{array}$ & - \\
\hline Tasty & - & $\begin{array}{c}3 \\
(12)\end{array}$ & $\begin{array}{c}1 \\
(25)\end{array}$ & - & $\begin{array}{c}3 \\
(60)\end{array}$ & $\begin{array}{c}2 \\
(8)\end{array}$ & $\begin{array}{c}5 \\
(20)\end{array}$ & $\begin{array}{c}3 \\
(50)\end{array}$ & $\begin{array}{c}5 \\
(38)\end{array}$ & $\begin{array}{c}5 \\
(20)\end{array}$ & $\begin{array}{c}3 \\
(50)\end{array}$ \\
\hline $\begin{array}{l}\text { Tasty }+ \text { less } \\
\text { production cost }\end{array}$ & - & - & - & - & - & - & $\begin{array}{c}3 \\
(13)\end{array}$ & - & & $\begin{array}{c}4 \\
(16)\end{array}$ & - \\
\hline $\begin{array}{l}\text { Testy + longer } \\
\text { durability of } \\
\text { boiled rice +less } \\
\text { nursing }\end{array}$ & $\begin{array}{c}1 \\
(35)\end{array}$ & $\begin{array}{c}5 \\
(20)\end{array}$ & - & $\begin{array}{c}2 \\
(50)\end{array}$ & - & - & - & - & - & - & - \\
\hline Fine rice & - & - & - & - & - & - & $\begin{array}{c}2 \\
(8)\end{array}$ & - & - & - & - \\
\hline Fine rice +tasty & - & - & - & - & - & - & $\begin{array}{c}2 \\
(8)\end{array}$ & - & - & - & $\begin{array}{c}3 \\
(50)\end{array}$ \\
\hline $\begin{array}{l}\text { Better yield +less } \\
\text { fertilizer use }\end{array}$ & - & $\begin{array}{c}5 \\
(20)\end{array}$ & - & - & - & - & - & - & - & - & \\
\hline Total & $\begin{array}{c}3 \\
(100)\end{array}$ & $\begin{array}{c}25 \\
(100)\end{array}$ & $\begin{array}{c}4 \\
(100)\end{array}$ & $\begin{array}{c}4 \\
(100)\end{array}$ & $\begin{array}{c}5 \\
(100)\end{array}$ & $\begin{array}{c}25 \\
(100)\end{array}$ & $\begin{array}{c}24 \\
(100)\end{array}$ & $\begin{array}{c}6 \\
(100)\end{array}$ & $\begin{array}{c}13 \\
(100)\end{array}$ & $\begin{array}{c}25 \\
(100)\end{array}$ & $\begin{array}{c}6 \\
(100)\end{array}$ \\
\hline
\end{tabular}

Note: Figures in the parentheses represent percentage. 
Bangladesh Rice J. 19(1):63 - 71, 2015

Table 12. Liking reasons for BRRI varieties in Dinajpur.

\begin{tabular}{|c|c|c|c|c|c|c|c|}
\hline Season & & & & & Boro & & \\
\hline Comment/Variety & BR11 & BR22 & BR1 & BR16 & $\begin{array}{c}\text { BRRI } \\
\text { dhan28 }\end{array}$ & $\begin{array}{c}\text { BRRI } \\
\text { dhan29 }\end{array}$ & $\begin{array}{l}\text { Hybrid } \\
\text { (Lal) }\end{array}$ \\
\hline Higher yield & $\begin{array}{c}2 \\
(50)\end{array}$ & $\begin{array}{c}2 \\
(33)\end{array}$ & - & $\begin{array}{c}13 \\
(52)\end{array}$ & $\begin{array}{c}8 \\
(54)\end{array}$ & $\begin{array}{c}2 \\
(67)\end{array}$ & $\begin{array}{c}11 \\
(69)\end{array}$ \\
\hline Tasty & $\begin{array}{c}1 \\
(25)\end{array}$ & $\begin{array}{c}2 \\
(33)\end{array}$ & $\begin{array}{c}2 \\
(67)\end{array}$ & - & $\begin{array}{c}3 \\
(20)\end{array}$ & - & $\begin{array}{c}5 \\
(31)\end{array}$ \\
\hline Tasty + less production cost & - & - & $\begin{array}{c}1 \\
(33)\end{array}$ & - & - & - & - \\
\hline $\begin{array}{l}\text { Tasty }+ \text { longer durability of boiled rice }+ \\
\text { less nursing }\end{array}$ & - & - & - & - & $\begin{array}{c}2 \\
(13)\end{array}$ & - & - \\
\hline Good quality rice & - & $\begin{array}{c}1 \\
(17)\end{array}$ & - & - & - & - & - \\
\hline Early rice variety & $\begin{array}{c}1 \\
(25)\end{array}$ & - & - & - & - & - & - \\
\hline Good for pop and puff (khai and muri) & - & - & - & $\begin{array}{c}8 \\
(32)\end{array}$ & - & - & - \\
\hline High grain weight & - & - & - & $\begin{array}{c}2 \\
(8)\end{array}$ & - & - & - \\
\hline Fine rice & - & - & - & - & - & $\begin{array}{c}1 \\
(33)\end{array}$ & - \\
\hline Fine rice +tasty & - & - & - & - & $\begin{array}{c}2 \\
(13)\end{array}$ & - & - \\
\hline $\begin{array}{l}\text { Fine rice }+ \\
\text { non-sticky }\end{array}$ & - & $\begin{array}{c}1 \\
(17)\end{array}$ & - & $\begin{array}{c}2 \\
(8)\end{array}$ & - & - & - \\
\hline Total & $\begin{array}{c}4 \\
(100) \\
\end{array}$ & $\begin{array}{c}6 \\
(100) \\
\end{array}$ & $\begin{array}{c}3 \\
(100)\end{array}$ & $\begin{array}{c}25 \\
(100) \\
\end{array}$ & $\begin{array}{c}15 \\
(100) \\
\end{array}$ & $\begin{array}{c}3 \\
(100) \\
\end{array}$ & $\begin{array}{c}16 \\
(100) \\
\end{array}$ \\
\hline
\end{tabular}

Note: Figures in the parentheses represent percentage.

Table 13. Liking reasons for BRRI varieties in Bhola.

\begin{tabular}{|c|c|c|c|c|c|c|c|c|c|c|c|c|}
\hline Season & & T. & $\operatorname{man}$ & & & & ro & & & & us & \\
\hline Comment/Variet & BR1 & BR3 & BR4 & Kaja & BRRI & BRRI & BRRI & Hybrid & BR & BR & BR2 & Kali \\
\hline $\mathrm{y}$ & 1 & 2 & 0 & 1 Sail & $\begin{array}{c}\text { dhan } 2 \\
8\end{array}$ & $\begin{array}{c}\text { dhan2 } \\
9\end{array}$ & $\begin{array}{l}\text { dhan } 4 \\
7\end{array}$ & -2 & 8 & 9 & 4 & $\begin{array}{c}\text { Hytt } \\
\text { a }\end{array}$ \\
\hline Higher yield & $\begin{array}{l}14 \\
(64)\end{array}$ & $\begin{array}{c}11 \\
(44)\end{array}$ & $\begin{array}{c}12 \\
(80)\end{array}$ & $\begin{array}{c}14 \\
(61)\end{array}$ & $\begin{array}{c}8 \\
(67)\end{array}$ & $\begin{array}{c}13 \\
(61)\end{array}$ & $\begin{array}{c}10 \\
(62)\end{array}$ & $\begin{array}{c}16 \\
(89)\end{array}$ & $\begin{array}{c}8 \\
(62)\end{array}$ & $\begin{array}{c}10 \\
(40)\end{array}$ & $\begin{array}{l}11 \\
(65)\end{array}$ & $\begin{array}{c}10 \\
(50)\end{array}$ \\
\hline Tasty & $\begin{array}{c}8 \\
(36)\end{array}$ & $\begin{array}{c}12 \\
(48)\end{array}$ & $\begin{array}{c}2 \\
(13)\end{array}$ & $\begin{array}{c}9 \\
(39)\end{array}$ & $\begin{array}{c}3 \\
(25)\end{array}$ & $\begin{array}{c}8 \\
(39)\end{array}$ & $\begin{array}{c}6 \\
(38)\end{array}$ & - & $\begin{array}{c}8 \\
(38)\end{array}$ & $\begin{array}{c}10 \\
(40)\end{array}$ & $\begin{array}{c}6 \\
(35)\end{array}$ & $\begin{array}{c}10 \\
(50)\end{array}$ \\
\hline $\begin{array}{l}\text { Tasty + less } \\
\text { production cost }\end{array}$ & - & - & $\begin{array}{c}1 \\
(7)\end{array}$ & - & - & - & - & - & & $\begin{array}{c}5 \\
(20)\end{array}$ & - & - \\
\hline $\begin{array}{l}\text { Testy + longer } \\
\text { durability of } \\
\text { boiled rice +less } \\
\text { nursing }\end{array}$ & - & - & - & - & $\begin{array}{c}1 \\
(8)\end{array}$ & - & - & $\begin{array}{c}2 \\
(11)\end{array}$ & - & - & - & - \\
\hline Fine rice & - & $\begin{array}{c}2 \\
(8)\end{array}$ & - & - & - & - & - & - & - & - & - & - \\
\hline Total & $\begin{array}{c}22 \\
(100)\end{array}$ & $\begin{array}{c}25 \\
(100)\end{array}$ & $\begin{array}{c}15 \\
(100)\end{array}$ & $\begin{array}{c}23 \\
(100)\end{array}$ & $\begin{array}{c}12 \\
(100)\end{array}$ & $\begin{array}{c}21 \\
(100)\end{array}$ & $\begin{array}{c}16 \\
(100)\end{array}$ & $\begin{array}{c}18 \\
(100)\end{array}$ & $\begin{array}{c}16 \\
(100)\end{array}$ & $\begin{array}{c}25 \\
(100)\end{array}$ & $\begin{array}{c}17 \\
(100)\end{array}$ & $\begin{array}{c}20 \\
(100)\end{array}$ \\
\hline
\end{tabular}

Note: Figures in the parentheses represent percentage.

Table 14. Reasons for liking varieties by the pure consumers in different locations.

\begin{tabular}{|c|c|c|c|c|}
\hline Reason & Gazipur & Dhaka & Dinajpur & Bhola \\
\hline Tasty & $\begin{array}{c}28 \\
(56)\end{array}$ & $\begin{array}{c}26 \\
(52)\end{array}$ & $\begin{array}{l}29 \\
(58)\end{array}$ & $\begin{array}{c}24 \\
(48)\end{array}$ \\
\hline Fine rice & $\begin{array}{c}10 \\
(20)\end{array}$ & $\begin{array}{c}12 \\
(24)\end{array}$ & $\begin{array}{l}11 \\
(22)\end{array}$ & $\begin{array}{c}9 \\
(18)\end{array}$ \\
\hline Fine rice + tasty & $\begin{array}{c}9 \\
(18)\end{array}$ & $\begin{array}{c}7 \\
(14)\end{array}$ & $\begin{array}{c}1 \\
(2)\end{array}$ & $\begin{array}{c}10 \\
(20)\end{array}$ \\
\hline Fine rice + non-sticky & $\begin{array}{c}3 \\
(6)\end{array}$ & $\begin{array}{c}5 \\
(10)\end{array}$ & $\begin{array}{c}9 \\
(18)\end{array}$ & $\begin{array}{c}7 \\
(14)\end{array}$ \\
\hline Total & $\begin{array}{c}50 \\
(100)\end{array}$ & $\begin{array}{c}50 \\
(100)\end{array}$ & $\begin{array}{c}50 \\
(100)\end{array}$ & $\begin{array}{c}50 \\
(100)\end{array}$ \\
\hline
\end{tabular}

Note: Figures in the parentheses represent percentage.CONCLUSIONS

70 Hossain et al 
Model analysis indicates that BR11, BR22 and BRRI dhan32 were more preferable in T. Aman season; BR16, BRRI dhan28 and BRRI dhan29 in Boro season and BR9, BR16 and BR20 in Aus season among the producers and producer-cumconsumers. Although, BRRI variety contributes about $90 \%$ of total production, it does not reflect in field label because BRRI varieties are sold in different brand names. As for example, BRRI dhan28 sales as Nizersail and BRRI dhan29 as Jhingasail and Miniket etc.

\section{REFERENCES}

Abdullahi, F A, M Zainalabidin and A L Ismail. 2011. The influence of socio-demographic factors and product attributes on attitudes toward purchasing special rice among Malaysian consumers. Int. Food Res. J. 18(3): 1135-1142.

Akaeze, Q O. 2010. Consumer Preference for imported rice in Nigeria - Perceived quality differences or habit persistence? Unpublished master thesis. Michigan State University.

Alauddin, M, C Tisdell. 1987. Trends and projections of Bangladeshi food production: an alternative viewpoint. Food Policy 12: 318-331.

Asaduzzaman, M, C Ringler, J Thurlow and S Alam. 2010. Investing in crop agriculture in Bangladesh for higher growth and productivity and adaptation to climate change. Bangladesh Food Security Investment Forum, Dhaka.

Azabagaoglu, M O and O Gaytancioglu. 2009. Analyzing consumer preference to different rice varieties in Turkey. Agricultura Tropica Et Subtropica, 42 (3): 118-125.

Basorun, J O. 2008. Analysis of the relationships of factors affecting rice consumption in a targeted region in Ekiti - State, Nigeria. Journal of Applied Quantitative Methods. 4.(2): 145-153.

BBS (Bangladesh Bureau of Statistics). 2009. Statistical Yearbook of Bangladesh. Ministry of Planning, Dhaka, Bangladesh.

BRRI (Bangladesh Rice Research Institute). 20010-11. Annual Report. BRRI, Dhaka, Bangladesh.

Diako, C, E Sakyi-Dawson, B Bediako-Amoa, F K Saalia and J T Manful. 2010. Consumer perceptions, knowledge and preferences for aromatic rice types in Ghana. Nature Sci. 8 (12): 12-19.

Galawat, F and M Yabe. 2010. Assesing consumer's preference for local rice in Brunei: An appplication of choice Model. J. ISSAAS. 16 (2): 104-115.

Gomez, A K, A A Gomez. 1983. Statistical Procedures for Agricultural Research. John Wiley and Sons, Inc, New York.

HIES (Household Income and Expenditure Survey). 2010. Bangladesh Bureau of Statistics. Government of Bangladesh, Dhaka.

Julian, B O. 1982. Consumer acceptance and processing characteristics of rice varieties. Paper presented at food conference of Singapore Institute of Food Science and Technology.

Kassali, R, R O Kareem, O Oluwasola and O M Ohaegbulam. 2010. Analysis of Demand for Rice in Ile Ife, Osun State, Nigeria. J. Sust. Develop. Africa. 12(2): 63-78.
Ladd, G W and V Suvannunt. 1976. A model of consumer good characteristics. Am. J. Agric. Econ. 58(1976): 504-510.

Larry, M. 2004. Consumer preference study and survey. A project completed in 2004, posted 2005. Iowa State University.

Laurian, J U, B O Juliano and C M Perez. 1985. Consumer demand for rice grain quality in Southeast Asia. Papers presented at the International Rice Research Conference, International Rice Research Institute (IRRI), Manila, Philippines.

Laurian, J U. 1986. Consumer demand for rice grain quality and returns to research for quality improvement in South Asia. Am. J. Agric. Econ. 68(3): 634-64.

Lançon, F, O Erenstein, S O Akande, S O Titilola, G Akpokodje and O O Ogundele. 2003. Imported rice retailing and purchasing in Nigeria: A survey. In the Nigerian Rice Economy in a competitive world: Constraints, opportunities and strategic choices. West Africa Rice Development Association (WARDA).

Suwannaporn, P and A Linnemann. 2008. Consumer preferences and buying criteria in rice: A study to identify market strategy for Thailand Jasmine Rice Export. J. Sensory Studies, 23: 1-13.

Tomlins, K I, J T Manful, P Larwer and L Hammond. 2005. Urban consumer preferences and sensory evaluation of locally produced and imported rice in West Africa. Food Quality Preference 16: 79-89.

Wong, L C Y, S A Emrus, M B Bashir and J Y S Tey. 2010. Malaysia Padi and Rice Industry: Applications of supply chain management approach. National Rice Conference. Swiss Garden Golf Resort, Lumut.

Yu, W H, M Alam, A Hassan, A S Khan, A C Ruane, C Rosenzweig, D C Major, J Thurlow. 2010. Climate change risk and food security in Bangladesh. EarthScan, London. 